PROCEEDINGS OF THE

AMERICAN MATHEMATICAL SOCIETY

Volume 126, Number 4, April 1998, Pages 1139-1142

S 0002-9939(98)04416-5

\title{
ON A CLASS OF RIESZ-FISCHER SEQUENCES
}

\author{
ROBERT M. YOUNG
}

(Communicated by Palle E. T. Jorgensen)

\begin{abstract}
In this note, we give necessary and sufficient conditions for a system of complex exponentials $\left\{e^{i \lambda_{n} t}\right\}$ to form a Riesz-Fischer sequence in $L^{2}(-A, A)$ for every positive number $A$. The result provides a significant strengthening of the sufficient conditions recently stated by R. M. Reid (1995).
\end{abstract}

\section{ON A CLASS OF RIESZ-FISCHER SEQUENCES}

The theory of nonharmonic Fourier series is concerned with the completeness, expansion, and interpolation properties of sets of complex exponentials $\left\{e^{i \lambda_{n} t}\right\}$ in $L^{p}(-A, A)$. Its origins, which are classical in spirit, lie in the celebrated works of Paley and Wiener [7] and Levinson [6]. In recent years, in response to the development of functional analysis and, in particular, to the growing interest in bases in Banach spaces, research in the area has flourished. New approaches to old problems have led to important advances in the theory.

In a recent issue of these Proceedings [8], the author proves the following generalization of the Riesz-Fischer theorem: If $\left\{\lambda_{n}\right\}$ is a sequence of real numbers whose differences are nondecreasing and satisfy

$$
\sum\left(\lambda_{n+1}-\lambda_{n}\right)^{-2}<\infty
$$

then the system $\left\{e^{i \lambda_{n} t}\right\}$ forms a Riesz-Fischer sequence in $L^{2}(-A, A)$ for every positive number $A$. This means that the moment problem

$$
\int_{-A}^{A} \phi(t) e^{-i \lambda_{n} t} d t=c_{n} \quad(n=1,2,3, \ldots)
$$

is solvable with $\phi$ in $L^{2}(-A, A)$ for any square summable sequence of scalars $\left\{c_{n}\right\}$. The proof is based on the theory of compact operators in Hilbert space together with the following elegant characterization of moment sequences due to Boas [3].

Theorem. If $\left\{f_{n}\right\}$ is a sequence of elements in a separable Hilbert space $H$, then the moment problem

$$
\left(f, f_{n}\right)=c_{n} \quad(n=1,2,3, \ldots)
$$

Received by the editors September 23, 1996.

1991 Mathematics Subject Classification. Primary 42A80.

Key words and phrases. Riesz-Fischer sequence, moment problem, upper uniform density.

(C)1998 American Mathematical Society 
is solvable with $f$ in $H$ for every square summable sequence of scalars $\left\{c_{n}\right\}$ if and only if the inequality

$$
m \sum\left|a_{n}\right|^{2} \leq\left\|\sum a_{n} f_{n}\right\|^{2}
$$

is valid for some positive constant $m$ and all finite sequences of scalars $\left\{a_{n}\right\}$.

Boas took $H$ to be $L^{2}(0,1)$. The general result given here was established independently by Bari [1], along with a number of other equivalent conditions (see also [10, pp. 155-157]).

The purpose of this note is to point out that condition (1) is far too restrictive. In fact, the following theorem provides the complete necessary and sufficient conditions. To simplify the notation, let us designate by $\Lambda$ an arbitrary sequence of real numbers and by $E(\Lambda)$ the corresponding system of complex exponentials. We say that $\Lambda$ is uniformly discrete if the distance between any two distinct points of $\Lambda$ exceeds some positive quantity. For such $\Lambda$, let $n^{+}(r)$ denote the largest number of points of $\Lambda$ to be found in an interval of length $r$. Then the limit

$$
D^{+}(\Lambda)=\lim _{r \rightarrow \infty} \frac{n^{+}(r)}{r},
$$

which always exists [2], is called the upper uniform density of $\Lambda$.

Theorem. The system $E(\Lambda)$ is a Riesz-Fischer sequence in $L^{2}(-A, A)$ for every positive number $A$ if and only if $\Lambda$ is uniformly discrete and has upper uniform density zero.

Proof. The necessity follows at once from a theorem of Landau [5], who showed that if $E(\Lambda)$ is a Riesz-Fischer sequence in $L^{2}(-A, A)$, then $\Lambda$ is uniformly discrete and $D^{+}(\Lambda) \leq A / \pi$.

The sufficiency is due to Beurling [2], who showed in essence that $E(\Lambda)$ is a Riesz-Fischer sequence in $L^{2}(-A, A)$ whenever $\Lambda$ is uniformly discrete and $D^{+}(\Lambda)<$ $A / \pi$.

Beurling's work was presented in a series of seminar lectures given in 1959-60 at the Institute for Advanced Study in Princeton, but was not published until 1989 when his collected works appeared. For an excellent account of these and related results, see [9].

Corollary. If $\Lambda$ is a sequence of real numbers for which

$$
\lambda_{n+1}-\lambda_{n} \rightarrow \infty \text { as } n \rightarrow \pm \infty,
$$

then $E(\Lambda)$ is a Riesz-Fischer sequence in $L^{2}(-A, A)$ for every positive number $A$.

Thus, under the assumption that successive differences of $\Lambda$ are nondecreasing, the condition of the corollary becomes both necessary and sufficient. This provides a dramatic relaxation of condition (1).

While Beurling's work lies fairly deep, the assertion of the corollary does not. It therefore seems instructive to provide a completely elementary proof.

Proof of the corollary. Fix $A>0$ and choose $N$ so large that

$$
\lambda_{n+1}-\lambda_{n} \geq \gamma>\pi / A
$$


whenever $|n|>N$. A well known result due Ingham [4] asserts that for some constant $m$, depending only on $\gamma$ and $A$, the inequality

$$
m \sum^{\prime}\left|a_{n}\right|^{2} \leq \int_{-A}^{A}\left|\sum^{\prime} a_{n} e^{i \lambda_{n} t}\right|^{2} d t
$$

holds for every finite sequence of scalars $\left\{a_{n}\right\}$. (Here, and in the statement below, the prime indicates that we are to disregard those $n$ for which $|n| \leq N$.) By the Boas-Bari theorem, the reduced system $E\left(\Lambda^{\prime}\right)$ is a Riesz-Fischer sequence in $L^{2}(-A, A)$.

Observe next that $E(\Lambda)$ must have infinite deficiency in $L^{2}(-A, A)$. This is an almost immediate consequence of Ingham's theorem. Let $M$ be a fixed but arbitrary positive integer. If $K$ is large enough, then we can replace

$$
\lambda_{-K}, \ldots, \lambda_{K}
$$

by

$$
\mu_{-K-M}, \ldots, \mu_{K+M},
$$

so that the resulting sequence, relabelled $\left\{\mu_{n}\right\}$, satisfies

$$
\mu_{n+1}-\mu_{n} \geq \mu>\pi / A \text {. }
$$

Thus the moment problem

$$
\int_{-A}^{A} \phi(t) e^{-i \mu_{n} t} d t= \begin{cases}1 & \text { if } n=0 \\ 0 & \text { if } n \neq 0\end{cases}
$$

is solvable with $\phi$ in $L^{2}(-A, A)$, and the system $\left\{e^{i \mu_{n} t}: n \neq 0\right\}$ is incomplete in $L^{2}(-A, A)$. But the completeness of a system of complex exponentials is unaffected if some exponent is replaced by another number (see, e.g., [10, p. 129]). Hence, the deficiency of $E(\Lambda)$ in $L^{2}(-A, A)$ is at least $2 M$, and, since $M$ was arbitrary, the deficiency must be infinite.

To complete the proof, we need only observe that an incomplete Riesz-Fischer sequence remains a Riesz-Fischer sequence whenever another exponential is adjoined. The details are straightforward and can be found in [9, p. 143].

\section{REFERENCES}

1. N. K. Bari, Biorthogonal systems and bases in Hilbert space, Učen. Zap. Mosk. Gos. Univ. 148, Mat. 4 (1951), 69-107. MR 14:289b

2. A. Beurling, "The Collected Works of Arne Beurling, Volume 2, Harmonic Analysis" (L. Carleson, P. Malliavin, J. Neuberger and J. Wermer, editors), pp. 341-365, Birkhäuser, Boston, 1989. MR 92k:01046b

3. R. P. Boas, Jr., A general moment problem, Amer. J. Math. 63 (1941), 361-370. MR 2:281d

4. A. E. Ingham, Some trigonometrical inequalities with applications to the theory of series, Math. Z. 41 (1936), 367-379.

5. H. J. Landau, Necessary density conditions for sampling and interpolation of certain entire functions, Acta Mathematica 117 (1967), 37-52. MR 36:5604

6. N. Levinson, "Gap and Density Theorems", Amer. Math. Soc. Colloq. Publ., Vol. 26, Amer. Math. Soc., New York, 1940. MR 2:180d

7. R.E.A.C. Paley and N. Wiener, "Fourier Transforms in the Complex Domain," Amer. Math. Soc. Colloq. Publ., Vol. 19, Amer. Math. Soc., New York, 1934.

8. R. M. Reid, A class of Riesz-Fischer sequences, Proc. Amer. Math. Soc. 123 (1995), 827-829. MR 95d:42012 
9. K. Seip, On the connection between exponential bases and certain related sequences in $L^{2}(-\pi, \pi)$, J. Funct. Anal. 130 (1995), 131-160. MR 96d:46030

10. R. M. Young, "An Introduction to Nonharmonic Fourier Series", Academic Press, New York, 1980. MR 81m: 42027

Department of Mathematics, Oberlin College, Oberlin, Ohio 44074

E-mail address: fyoungb@ocvaxa.cc.oberlin.edu 